PROCEEDINGS OF THE

AMERICAN MATHEMATICAL SOCIETY

Volume 140, Number 3, March 2012, Pages 1105-1109

S 0002-9939(2011)10966-3

Article electronically published on July 26, 2011

\title{
HANDLE NUMBER ONE LINKS AND GENERALIZED PROPERTY $R$
}

\author{
MICHAEL J. WILLIAMS
}

(Communicated by Daniel Ruberman)

\begin{abstract}
It is shown that if the exterior of a link $L$ in the three sphere admits a genus 2 Heegaard splitting, then $L$ has Generalized Property $R$.
\end{abstract}

\section{INTRODUCTION}

A knot $K$ in the three sphere $\mathbb{S}^{3}$ is said to have Property $\boldsymbol{R}$ if $K$ is the unknot, or 0 -framed Dehn surgery on $K$ does not yield $\mathbb{S}^{1} \times \mathbb{S}^{2}$. Gabai [2] proved that every knot in $\mathbb{S}^{3}$ has Property $R$. A generalization to links is the following:

Generalized Property $\mathbf{R}$ Conjecture. If Dehn surgery on an n-component framed link $L \subset \mathbb{S}^{3}$ yields $\#_{n}\left(\mathbb{S}^{1} \times \mathbb{S}^{2}\right)$, then there is a sequence of handleslides which converts $L$ to the $n$-component 0 -framed unlink.

Recall that the handleslide is one of the basic operations of Kirby calculus in which a framed link component gets replaced by its band connected sum with the framing of another framed component; see [4, Chapter 4] for more details. All the framings on a link $L$ satisfying the hypothesis of Generalized Property $R$ must be the 0 -framing, and the components of $L$ must be algebraically unlinked; this is due to classical homological arguments (see [3, Proposition 2.2]). We will say that a link $L$ has Generalized Property $\boldsymbol{R}$ if $L$ satisfies the the Generalized Property $R$ Conjecture.

Only a few (easily describable) infinite families of links are known to have Generalized Property $R$. These include the tunnel number one links [3, Proposition 3.1] and the 2-component links which have at least one unknotted component [3] Proposition 3.2].

Our main result below is inspired by [3, Proof of Proposition 3.1], and resolves the Generalized Property $R$ Conjecture for all links whose exteriors admit genus 2 Heegaard splittings.

Main Theorem. Suppose that $L \subset \mathbb{S}^{3}$ is an n-component link $(n>1)$ whose exterior admits a genus 2 Heegaard splitting. Then $L$ has Generalized Property $R$. Furthermore, if 0 -framed surgery on $L$ yields $\#_{n}\left(\mathbb{S}^{1} \times \mathbb{S}^{2}\right)$, then $n=2$ and the components of $L$ are unknots.

Received by the editors October 20, 2009 and, in revised form, December 22, 2009 and December $22,2010$.

2010 Mathematics Subject Classification. Primary 57M25, 57M27.

The research was supported by a University of California President's Postdoctoral Fellowship and the UC Santa Barbara Department of Mathematics.

(c) 2011 American Mathematical Society Reverts to public domain 28 years from publication 1105 
Indeed, our main theorem generalizes Generalized Property $R$ for tunnel number one links to handle number one links; see Section 2 for definitions. For example, any link consisting of a "couple of 2-bridge knots" (as defined in [7, Section 3.1]) has handle number one but not tunnel number one; such links also have linking number zero. So our main theorem implies that 0-framed surgery on a "couple of 2-bridge knots" can never yield $\#_{2}\left(\mathbb{S}^{1} \times \mathbb{S}^{2}\right)$.

Part of the significance of the Generalized Property $R$ Conjecture lies in the realm of smooth 4-dimensional handle structures. The closed 3-manifold $M$ obtained by integral Dehn surgery on an $n$-component link $L$ in $\mathbb{S}^{3}$ is the boundary of a compact, orientable, simply connected 4 -manifold $X$ obtained by attaching 2-handles to the standard smooth 4-ball $B^{4}$ along $L$ with framings given by the Dehn surgery slopes; see [4. Chapters 4 and 5] for details. If $M=\#_{n}\left(\mathbb{S}^{1} \times \mathbb{S}^{2}\right)$, then the $\mathbb{S}^{2}$ factors of the $\mathbb{S}^{1} \times \mathbb{S}^{2}$ summands serve as attaching regions for attaching 3-handles to $X$; this yields a smooth homotopy 4-ball $X^{\prime}$ with $\partial X^{\prime}=\mathbb{S}^{3}$. If $L$ has Generalized Property $R$, then the 2-handles and 3-handles can be arranged to cancel in pairs, giving a standard handle structure on $B^{4}$. Hence $X^{\prime}$ is diffeomorphic to $B^{4}$, preventing $X^{\prime}$ from generating a counterexample to the Smooth Poincaré Conjecture in dimension 4. For more information, see [8, Section 2] and [1, Section 3].

Our notation for Dehn surgery will be the following. Given a link $L \subset \mathbb{S}^{3}$ with components $L_{1}, \ldots, L_{n}$ and corresponding framings $r_{1}, \ldots, r_{n} \in \mathbb{Z}$, let $L\left(r_{1}, \ldots, r_{n}\right)$ denote the 3-manifold obtained by Dehn surgery on the framed link $L$. If all the $r_{i}$ are equal to a particular $r \in \mathbb{Z}$, we will refer to $L$ as an $r$-framed link.

\section{HANDLE NUMBER ONE LINKS}

A system of unknotting tunnels for a link $L$ in $\mathbb{S}^{3}$ is a collection of disjoint properly embedded $\operatorname{arcs} \gamma_{1}, \ldots, \gamma_{k}$ in the link exterior $E(L)$ with the property that drilling $\bigcup \gamma_{i}$ out of $E(L)$ yields a handlebody. The tunnel number $\boldsymbol{t}(\boldsymbol{L})$ for a link $L$ is the least number required in a system of unknotting tunnels for $L$. A tunnel system for $E(L)$ induces a Heegaard splitting for $E(L)$ in which all of the boundary components of $E(L)$ lie on one side of the splitting surface. Only the unknot satisfies $t(L)=0$.

Now suppose that $L$ is a link in $\mathbb{S}^{3}$ with tunnel number one (i.e. $t(L)=1$ ). Then $n \leq 2$, and we see that $E(L)$ is obtained by attaching a 2-handle to a genus 2 handlebody $H$ along a simple closed curve $\alpha \subset \partial H$. Note that $n=2$ if and only if $\alpha$ separates $\partial H$.

A system of unknotting handles for a link $L$ in $\mathbb{S}^{3}$ is a collection of disjoint properly embedded arcs $\gamma_{1}, \ldots, \gamma_{k}$ in the exterior $E(L)$ with the property that drilling $\bigcup \gamma_{i}$ out of $E(L)$ yields a compression body; this clearly generalizes a system of unknotting tunnels. The handle number $\boldsymbol{h}(\boldsymbol{L})$, following [9, for a link $L$ is the least number required in a system of unknotting handles for $L$. We note that $h(L) \leq t(L)$. A handle system for $E(L)$ induces a Heegaard splitting for $E(L)$ in which the boundary components of $E(L)$ do not necessarily lie on one side of the splitting surface. Only the unknot and the Hopf link satisfy $h(L)=0$.

Now suppose that $L$ is a link in $\mathbb{S}^{3}$ with handle number one (i.e. $h(L)=1$ ). Then $n \leq 4$, and we see that $E(L)$ is obtained by attaching a 2-handle to a compression body $W$ along a simple closed curve $\alpha \subset \partial_{+} W$ where $\partial_{+} W$ has genus 2 . 


\section{Proof of Main Theorem}

The proof will involve fundamental group calculations. In order to keep our notation as simple as possible, we will adopt the following convention: if $M$ is a connected 3-manifold with basepoint $p \in M$ and if $\gamma \subset M$ is a loop, then we will also let $\gamma$ denote the homotopy class of $\gamma$ in $\pi_{1}(M, p)$, possibly under an appropriate change-of-basepoint isomorphism when $p \notin \gamma$. If $G$ is a group and $S \subset G$, let $\langle\langle S\rangle\rangle$ denote the normal closure of $S$ in $G$.

Proof of Main Theorem. Let $L \subset \mathbb{S}^{3}$ be an $n$-component link $n>1$ whose exterior $E(L)$ admits a genus 2 Heegaard splitting. Therefore, any Dehn surgery on $L$ admits a genus 2 Heegaard splitting. We also observe that $n \leq 4$. By additivity of Heegaard genus under connected sums, we see that $\#{ }_{m}\left(\mathbb{S}^{1} \times \mathbb{S}^{2}\right)$ has Heegaard genus $m$ for any $m \in \mathbb{N}$. By assumption, 0 -framed Dehn surgery on $L$ yields $\#_{n}\left(\mathbb{S}^{1} \times \mathbb{S}^{2}\right)$, so $n=2$.

Let $L_{1}$ and $L_{2}$ be the components of $L$ with respective regular neighborhoods $N\left(L_{1}\right)$ and $N\left(L_{2}\right)$. So $L$ has exterior $E(L)=\overline{\mathbb{S}^{3}-\left(N\left(L_{1}\right) \cup N\left(L_{2}\right)\right)}$. Let $\partial_{1}$ and $\partial_{2}$ be the boundary components of $E(L)$, so that $\partial_{i}$ corresponds to $L_{i}$. Let $m_{i}, l_{i}$ be a standard oriented meridian-longitude pair for $\partial_{i}$ for $i=1,2$.

Now, if $L$ has tunnel number one, then we are done by [3, Proposition 3.1]. So we may assume that there is a genus 2 Heegaard splitting of $E(L)$ which separates at least two boundary components. Hence the exterior of $L$ can be realized as $E(L)=$ $W \cup_{\alpha}\left(2\right.$-handle) where $W$ is a compression body with $\partial_{-} W=\partial_{1}$, genus $\left(\partial_{+} W\right)=2$, and $\alpha$ is a nonseparating curve on $\partial_{+} W$. Note that $W$ is obtained by attaching a 1 -handle to (torus) $\times[0,1]$ on (torus) $\times\{1\}$. If we slide the endpoints of the core of the 1-handle together, we obtain a simple closed curve $\tau$ which meets (torus) $\times\{1\}$ in one point; we push $\tau$ toward $\partial_{-} W=$ (torus) $\times\{0\}=\partial_{1}$ to meet it in a point $p$. Give $\tau$ an arbitrary orientation and situate the curves $m_{1}$ and $l_{1}$ on $\partial_{1}$ so that $m_{1} \cap l_{1}=p$; see Figure 1. It is now clear that

$$
\begin{aligned}
& \pi_{1}(W, p) \cong\left\langle m_{1}, l_{1}, \tau: m_{1} l_{1}=l_{1} m_{1}\right\rangle \cong(\mathbb{Z} \oplus \mathbb{Z}) * \mathbb{Z}, \\
& \pi_{1}(E(L), p) \cong \pi_{1}(W, p) /\langle\langle\alpha\rangle\rangle, \text { and } \\
& \pi_{1}(L(0,0), p) \cong \pi_{1}(E(L), p) /\left\langle\left\langle l_{1}, l_{2}\right\rangle\right\rangle \cong \#_{2}\left(\mathbb{S}^{1} \times \mathbb{S}^{2}\right) \cong \mathbb{Z} * \mathbb{Z} .
\end{aligned}
$$

To simplify notation, the basepoint $p \in W \subset E(L) \subset L(0,0)$ will be suppressed in fundamental group expressions for the remainder of the proof. We have epimorphisms

$$
\pi_{1}(W) \stackrel{\phi}{\longrightarrow} \pi_{1}(E(L)) \stackrel{\rho}{\longrightarrow} \pi_{1}(L(0,0)),
$$

where $\phi$ is reduction mod $\langle\langle\alpha\rangle\rangle$ and $\rho$ is reduction $\bmod \left\langle\left\langle l_{1}, l_{2}\right\rangle\right\rangle$. Consider another sequence of epimorphisms,

$$
\pi_{1}(W) \stackrel{\phi}{\longrightarrow} \pi_{1}(E(L)) \stackrel{\rho_{0}}{\longrightarrow} \pi_{1}(E(L)) /\left\langle\left\langle l_{1}\right\rangle\right\rangle,
$$

where $\rho_{0}$ is reduction mod $\left\langle\left\langle l_{1}\right\rangle\right\rangle$. Note that the epimorphism $\rho$ factors through $\rho_{0}$; that is, there is an epimorphism

$$
\pi_{1}(E(L)) /\left\langle\left\langle l_{1}\right\rangle\right\rangle \stackrel{\rho_{1}}{\longrightarrow} \pi_{1}(L(0,0)),
$$




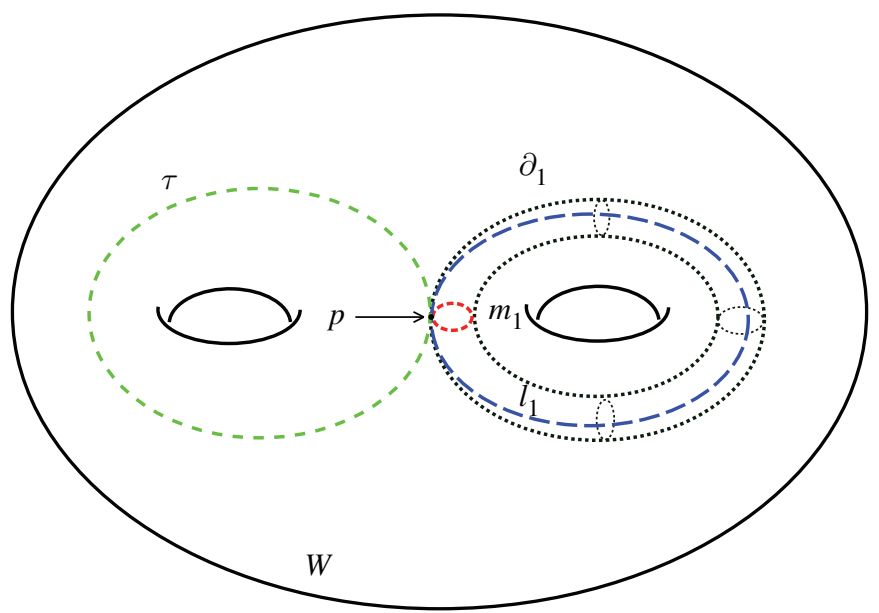

Figure 1 . The compression body $W$ with curves $m_{1}$ and $l_{1}$ on $\partial_{-} W=\partial_{1}$ and the "tunnel curve" $\tau$. The curves $m_{1}, l_{1}$, and $\tau$ all meet the basepoint $p$. Note that $\tau \cup \partial_{1}$ forms a spine for $W$.

namely reduction $\bmod \left\langle\left\langle l_{2}\right\rangle\right\rangle$, so that $\rho=\rho_{1} \circ \rho_{0}$. Since $\left(\rho_{0} \circ \phi\right)\left(l_{1}\right)=1$, the epimorphism $\phi$ naturally descends to an epimorphism

$$
\pi_{1}(W) /\left\langle\left\langle l_{1}\right\rangle\right\rangle \stackrel{\phi^{\prime}}{\longrightarrow} \pi_{1}(E(L)) /\left\langle\left\langle l_{1}\right\rangle\right\rangle .
$$

Note that $W \cup_{l_{1}}\left(2\right.$-handle) is a punctured genus 2 handlebody, so $\pi_{1}(W) /\left\langle\left\langle l_{1}\right\rangle\right\rangle \cong$ $\mathbb{Z} * \mathbb{Z}$. In summary, we have a commutative diagram of epimorphisms:

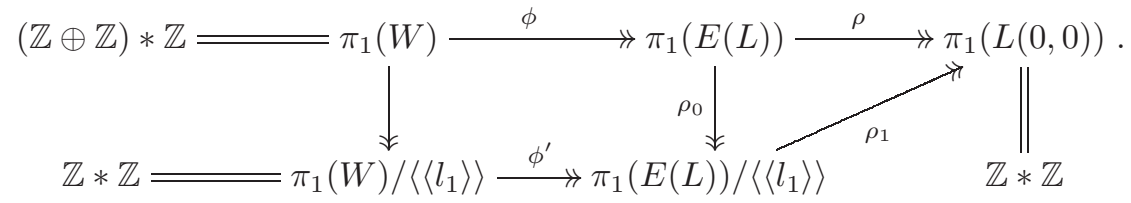

Since the group $\mathbb{Z} * \mathbb{Z}$ is Hopfian (every self-epimorphism is an isomorphism), we must have all isomorphisms in the sequence

$$
\mathbb{Z} * \mathbb{Z}=\pi_{1}(W) /\left\langle\left\langle l_{1}\right\rangle\right\rangle \stackrel{\phi^{\prime}}{\longrightarrow} \pi_{1}(E(L)) /\left\langle\left\langle l_{1}\right\rangle\right\rangle \stackrel{\rho_{1}}{\longrightarrow} \pi_{1}(L(0,0))=\mathbb{Z} * \mathbb{Z} .
$$

We now see that $\pi_{1}\left(E_{1}\right) \cong \pi_{1}(E(L)) /\left\langle\left\langle l_{1}\right\rangle\right\rangle \cong \mathbb{Z} * \mathbb{Z}$, where $E_{1}$ is the 3 -manifold obtained from $E(L)$ by Dehn filling along $l_{1} \subset \partial \mathrm{N}\left(L_{1}\right)$. By [5, Theorem 5.2], the Prime Decomposition Theorem, and the fact that genus 23 -manifolds satisfy the Poincaré Conjecture (see [6]), we conclude that

$$
E_{1} \cong\left(\mathbb{S}^{1} \times D^{2}\right) \#\left(\mathbb{S}^{1} \times \mathbb{S}^{2}\right) .
$$

Furthermore, Dehn filling on $E_{1}$ along $l_{2} \subset \partial \mathrm{N}\left(L_{2}\right)$ gives $L(0,0) \cong\left(\mathbb{S}^{1} \times \mathbb{S}^{2}\right) \#\left(\mathbb{S}^{1} \times\right.$ $\left.\mathbb{S}^{2}\right)$, so $l_{2}$ corresponds to a meridian curve in the $\mathbb{S}^{1} \times D^{2}$ connected-summand of $E_{1}$. Note that this is just the topological realization of the epimorphisms $\rho_{0}$ and $\rho_{1}$. It follows that $m_{2}$ generates a free factor of $\pi_{1}\left(E_{1}\right)$. Hence $\pi_{1}\left(E_{1}\right) /\left\langle\left\langle m_{2}\right\rangle\right\rangle \cong \mathbb{Z}$. 
Let $L_{1}(0)$ denote the 3 -manifold obtained by 0 -framed Dehn surgery on the knot $L_{1}$. We see that

$$
\begin{aligned}
\pi_{1}\left(L_{1}(0)\right) & \cong \pi_{1}(E(L)) /\left\langle\left\langle l_{1}, m_{2}\right\rangle\right\rangle \\
& \cong\left(\pi_{1}(E(L)) /\left\langle\left\langle l_{1}\right\rangle\right\rangle\right) /\left\langle\left\langle m_{2}\right\rangle\right\rangle \\
& \cong \pi_{1}\left(E_{1}\right) /\left\langle\left\langle m_{2}\right\rangle\right\rangle \\
& \cong \mathbb{Z} .
\end{aligned}
$$

By [5, Theorem 5.2], we have that $L_{1}(0) \cong \mathbb{S}^{1} \times \mathbb{S}^{2}$. Applying [2, Corollary 8.3] establishes that $L_{1}$ must be the unknot. We can similarly establish that $L_{2}$ is also the unknot; this is accomplished by simply interchanging the roles of $L_{1}$ and $L_{2}$ throughout the proof. Finally, the result [3. Proposition 3.2] asserts that any 2component link containing an unknot has Generalized Property $R$. This completes the proof.

\section{ACKNOWLEDGEMENTS}

The author would like to thank Martin Scharlemann and Abigail Thompson for helpful and inspiring conversations. The author also thanks the referee for useful suggestions.

\section{REFERENCES}

[1] Michael Freedman, Robert Gompf, Scott Morrison, and Kevin Walker, Man and machine thinking about the smooth 4-dimensional Poincaré conjecture, Quantum Topol. 1 (2010), no. 2, 171-208. MR2657647

[2] David Gabai, Foliations and the topology of 3-manifolds. III, J. Differential Geom. 26 (1987), no. 3, 479-536. MR910018 (89a:57014b)

[3] Robert E Gompf, Martin Scharlemann, and Abigail Thompson, Fibered knots and potential counterexamples to the Property $2 R$ and Slice-Ribbon Conjectures, Geom. Topol. 14 (2010), no. 4, 2305-2347. MR 2740649

[4] Robert E. Gompf and András I. Stipsicz, 4-manifolds and Kirby calculus, Graduate Studies in Mathematics, vol. 20, American Mathematical Society, Providence, RI, 1999. MR 1707327 (2000h:57038)

[5] John Hempel, 3-Manifolds, Princeton University Press, Princeton, NJ, 1976, Ann. of Math. Studies, No. 86. MR0415619 (54:3702)

[6] John W. Morgan and Hyman Bass (eds.), The Smith conjecture, Pure and Applied Mathematics, vol. 112, Academic Press Inc., Orlando, FL, 1984, papers presented at the symposium held at Columbia University, New York, 1979. MR758459 (86i:57002)

[7] Yoav Moriah and Eric Sedgwick, Closed essential surfaces and weakly reducible Heegaard splittings in manifolds with boundary, J. Knot Theory Ramifications 13 (2004), no. 6, 829843. MR2088748 (2005i:57023)

[8] Martin Scharlemann, Generalized property $R$ and the Schoenflies conjecture, Comment. Math. Helv. 83 (2008), no. 2, 421-449. MR2390052 (2008m:57058)

[9] Eric Sedgwick, Genus two 3-manifolds are built from handle number one pieces, Algebr. Geom. Topol. 1 (2001), 763-790 (electronic). MR1875617 (2002k:57051)

Department of Mathematics, University of California, Riverside, California 92521

E-mail address: mwilliam@ucr.edu 